\title{
Comparative study of flavor in cholesterol-removed Gouda cheese and Gouda cheese during ripening
}

\author{
H. J. Jung, ${ }^{*}$ P. Ganesan, ${ }^{*}$ S. J. Lee, $†$ and H. S. Kwak* \\ *Department of Food Science and Technology, and \\ †Department of Food Service Management, Sejong University, Seoul 143-747, Korea
}

\begin{abstract}
This study was performed to compare the flavor compounds of cholesterol-removed Gouda cheese (CRGC) and those of Gouda cheese (control) during ripening. The CRGC was made using milk treated with crosslinked $\beta$-cyclodextrin ( $\beta$-CD). The solid-phase microextraction (SPME) method was used to extract flavor compounds from Gouda cheese. In both CRGC and control cheese, 31 flavor compounds were identified, including 6 free fatty acids, 5 esters, 5 ketones, 1 aldehyde, 3 lactones, 5 alcohols, and 6 miscellaneous compounds. Free fatty acids were the most abundant flavor compounds quantified in CRGC and control cheese. In the early stage of ripening, concentrations of flavor compounds in CRGC and control cheese were 16.42 and $10.38 \mathrm{mg} / \mathrm{kg}$, respectively. At $6 \mathrm{mo}$, they increased to 40.90 and $67.89 \mathrm{mg} / \mathrm{kg}$, respectively. A group of esters was the second abundant flavor compound in CRGC and control cheese. At the initial stage of ripening, total concentrations of esters were 12.94 (CRGC) and 10.95 $\mathrm{mg} / \mathrm{kg}$ (control) and they increased to 22.73 (CRGC) and $27.68 \mathrm{mg} / \mathrm{kg}$ (control). Total concentrations of ketones were 1.96 (CRGC) and $6.49 \mathrm{mg} / \mathrm{kg}$ (control) at the initial stage of ripening. After 6 mo of ripening, total concentrations reached 11.32 (CRGC) and 52.43 $\mathrm{mg} / \mathrm{kg}$ (control). In the case of the lactones, at the early stage of ripening, total concentrations of CRGC and control cheese were 0.63 and $0.84 \mathrm{mg} / \mathrm{kg}$, respectively, and then increased to 1.73 (CRGC) and $3.25 \mathrm{mg} / \mathrm{kg}$ (control) at the end of ripening. Based on the results of this study, the flavor compounds of CRGC and control showed slightly different profiles during ripening.
\end{abstract}

Key words: flavor compound, Gouda cheese, cholesterol-removed Gouda cheese

\section{INTRODUCTION}

Yellowish Gouda cheese is one of the Dutch-type cheese varieties produced worldwide from bovine, ovine,

Received April 20, 2012.

Accepted January 2, 2013.

${ }^{1}$ Corresponding author: kwakhs@sejong.ac.kr or caprine milk (Walstra et al., 1993a,b). Ripening of Gouda cheese takes 1 to 20 mo and it can be consumed based on the flavor preference at various stages of maturity (Walstra et al., 1993a,b). Fat plays a physical role in the cheese flavoring by acting as a solvent for the various flavor-forming reactions (Wijesundera and Drury, 1999). Lipolysis increases the fat-derived compounds, which favors the cheesy flavors in Gouda cheeses (Kinsella, 1975). The fat-derived compounds include FFA, esters, lactones, and ketones, and the formation of cheese flavor is limited by some other parameters such as starter cultures, nonstarter lactic acid bacteria, or nonstarter lactic acid bacteria flora in the cheese plant, cheesemaking technique, and content of fat or salt (Chamba and Perreard, 2002; Mallatou et al., 2003).

Trends toward healthier eating habits provide increasing interest in low-fat products. However, lowering fat becomes a great concern in Gouda cheese making, which greatly affects the flavor derived from the fat components. Therefore, lowering cholesterol is an alternative in the production of Gouda cheese. Very few studies have investigated the flavor chemistry effect of cholesterol reduction in some cheeses. Jeon et al. $(2012 \mathrm{a}, \mathrm{b})$ reported that cholesterol removal in whole-milk powder cream cheese and cream cheese was almost identical. Further, volatile and short-chain FFA between the cheeses with and without cholesterol removed were similar. However, no information is available about the effects of cholesterol removal on the volatile profile of Gouda cheese. Because most consumers are not willing to sacrifice the flavor of fat or fatreduced Gouda cheese, it is crucial to understand the effect of cholesterol reduction on Gouda cheese volatile profiles. Therefore, the main purpose of this study was to determine the effect of cholesterol reduction on the flavor profiles of Gouda cheese.

\section{MATERIALS AND METHODS}

\section{Materials}

Raw milk for manufacture Gouda cheese was purchased from Youngduck Farm (Gyeonggi-do, Korea). 
Vials, Teflon-coated rubber septa, and aluminum caps and a manual solid-phase microextraction (SPME) fiber holder unit (50/30 $\mu \mathrm{m}$ divinylbenzene/Carboxen/ polydimethylsiloxane) were purchased from Supelco Inc. (Bellefonte, PA). Isoamyl alcohol and $\mathrm{H}_{2} \mathrm{SO}_{4}$ were purchased from Yakuri Pure Chemicals Co. Ltd. (Osaka, Japan). Commercial $\beta$-cyclodextrin ( $\beta$-CD; purity 99.1\%) was obtained from Nihon Shokuhin Cako Co. Ltd. (Osaka, Japan). A frozen concentrated direct vat set mesophilic lactic acid starter culture (CHN 11) and rennet (Stamix 1150) were purchased from Chr. Hansen's Laboratory (Copenhagen, Denmark). As standard chemicals, cholesterol, $5 \alpha$-cholestane, n-alkane, undecane butanoic acid, hexanoic acid, octanoic acid, and decanoic acid were purchased from Sigma (St. Louis, $\mathrm{MO}$ ) and all solvents were gas chromatography grade.

\section{Determination of Milk Fat}

Determination of milk fat in bulk milk and cream was performed using the method of Gerber (BSI, 1969). In a butyrometer, $10 \mathrm{~mL}$ of $\mathrm{H}_{2} \mathrm{SO}_{4}(92 \% \mathrm{wt} / \mathrm{vol}), 11 \mathrm{~mL}$ of milk, and $1 \mathrm{~mL}$ of isoamyl alcohol were added, and then the butyrometer was capped with a rubber cap and shaken vigorously until all particles were melted. The butyrometer was then placed in a water bath set at $60^{\circ} \mathrm{C}$ for $15 \mathrm{~min}$ and centrifuged at $166 \times g$ for $5 \mathrm{~min}$. Separated milk fat was determined using a divider.

\section{Preparation of Cross-Linked $\beta-C D$}

The cross-linked $\beta$-CD was made according to the method of Han et al. (2007). Eighty grams of $\beta$-CD powder was dissolved in $100 \mathrm{~mL}$ of distilled water and placed on a hot plate magnetic stirrer (Misung Scientific Co. Ltd., Seoul, Korea) with constant stirring at room temperature for $2 \mathrm{~h}$. Then, $5 \mathrm{~g}$ of adipic acid was added and the $\mathrm{pH}$ was adjusted to $\mathrm{pH} 10$ using $1 \mathrm{~N} \mathrm{NaOH}$. The $\beta$-CD solution was stirred again with constant agitation at room temperature for $90 \mathrm{~min}$. Finally, the solution was adjusted to $\mathrm{pH} 5$ using acetic acid. To recover the cross-linked $\beta$-CD powder, the $\beta$-CD solution was filtered through Whatman paper no. 2 and washed 3 times with distilled water. Then, the collected powder was dried at $60^{\circ} \mathrm{C}$ in a Lab-Line mechanical convection oven (O-SUNG Scientific Co., Seoul, South Korea) for $20 \mathrm{~h}$ and passed through a $100-$ mesh $(150-\mu \mathrm{m})$ sieve.

\section{Milk Treatment and Cholesterol Removal}

Bulk raw milk (milk fat: $3.8 \%$ ) was pasteurized at $65^{\circ} \mathrm{C}$ for $30 \mathrm{~min}$ and cream was separated at $50^{\circ} \mathrm{C}$ using a cream separator (CE Elecrem, Vanves, France). Separated cream was stirred with cross-linked $\beta-C D$
(10\% of amount of cream) at 1,400 rpm with a blender (Tops; Misung Co., Seoul, Korea) in a thermostatic water bath at $40^{\circ} \mathrm{C}$ for $30 \mathrm{~min}$. To separate out cholesterol-entrapped $\beta-C D$ from the cream, the cream was centrifuged at $166 \times g$ for $10 \mathrm{~min}$. The food-grade cholesterol-removed cream was reconstituted with skim milk at $70.4 \mathrm{~kg} / \mathrm{cm}^{2}$ (HC 5000; Microfluidics Corp., Newton, MA; Seon, et al., 2009).

\section{Manufacture of Gouda Cheese}

Gouda cheese was produced using the method described by Kosikowski and Mistry (1997). The cholesterol removed milk (115 L) was warmed to 32 to $33^{\circ} \mathrm{C}$. A frozen concentrated direct vat set mesophilic lactic acid starter culture (CHN 11; strain Lactococcus lactis) was added to the milk. After $90 \mathrm{~min}, 23 \mathrm{~mL}$ of rennet (diluted 1:3) (Chr. Hansen's Laboratory) was added to the milk sample. Milk was then stirred quickly for 3 min and set to coagulate at 32 to $33^{\circ} \mathrm{C}$ for $45 \mathrm{~min}$ and the curd was cut [titratable acidity $=0.12 \%$ (wt $/ \mathrm{wt}$ ) lactic acid] to a size of $1 \times 1 \times 1 \mathrm{~cm}$, and the curd-whey mixture was gradually stirred. After stirring, the curdwhey mixture temperature was gradually increased to $37^{\circ} \mathrm{C}$. After $30 \mathrm{~min}$ of cooking, whey $(10 \%$ of the volume of the milk) was partially removed and replaced by an equal volume with water of the same temperature to reduce lactose and to control lactic acid production during the manufacturing process. The curd-whey mixture was stirred for $30 \mathrm{~min}$ at the cooking temperature. After draining, the curd was transferred to the small vat for prepress. The prepress was done for $30 \mathrm{~min}$ at $150 \mathrm{kPa}$. The curds were put into wheel-shaped plastic molds. The curds were pressed at $300 \mathrm{kPa}$ for $20 \mathrm{~h}$ with $2 \sim 3 \mathrm{~h}$ interval. The next morning, the fresh cheese was brined in a $20 \%$ (wt/wt) brine solution at $14^{\circ} \mathrm{C}$ for $8 \mathrm{~h}$. After draining off from the brine and drying the cheese, it was ripened at $14^{\circ} \mathrm{C}$ for 6 mo at a relative humidity of $85 \%$. Samples for analysis were periodically taken at $0,1,2,3,4,5$, and 6 mo of ripening. Both cheeses were made in duplicate in 2 batches.

\section{Chemical Composition and Yield of Cheese}

Moisture and protein were analyzed using the methods of the Association of Official Analytical Chemists (AOAC International, 2000). Fat content in cheese was determined by method described by the British Standards Institution (BSI, 1969) using a milk butyrometer and yield was calculated as the weight of cheese $\times 100 /$ weight of milk.

\section{Extraction of Cholesterol}

Cholesterol was quantified according to the modified method of Adams et al. (1986) using gas chromatogra- 
phy. One gram of ground cheese sample was placed in a screw-capped glass tube $(15 \times 180 \mathrm{~mm})$, and 1,000 mg/ $\mathrm{kg}$ of $5 \alpha$-cholestane was added as an internal standard. The sample was saponified at $60^{\circ} \mathrm{C}$ for $30 \mathrm{~min}$ with $5 \mathrm{~mL}$ of $2 \mathrm{M} \mathrm{KOH} \mathrm{(wt/vol).} \mathrm{After} \mathrm{cooling} \mathrm{to} \mathrm{room}$ temperature, cholesterol was extracted with $5 \mathrm{~mL}$ of hexane. This process was repeated 4 times. The hexane layers were transferred to a round-bottomed flask and dried under vacuum. The extract was redissolved in 1 $\mathrm{mL}$ of hexane and was stored at $-20^{\circ} \mathrm{C}$ until analysis. All experiments were performed in triplicate.

\section{Determination of Cholesterol}

Cholesterol was determined on a silica fused capillary column (HP-5, $30 \mathrm{~m} \times 0.32-\mathrm{mm}$ i.d. $\times 0.25-\mu \mathrm{m}$ thickness) using a Donam gas chromatograph (Donam Instruments Inc., Seoul, Korea) equipped with a flameionization detector (FID). The temperatures of the injector and detector were 270 and $300^{\circ} \mathrm{C}$, respectively. The oven temperatures were programmed from 200 to $300^{\circ} \mathrm{C}$ at $10^{\circ} \mathrm{C} / \mathrm{min}$ and held for $20 \mathrm{~min}$. Nitrogen was used as a carrier gas at a flow rate of $2 \mathrm{~mL} / \mathrm{min}$, with a split ratio of 1:50. Quantification of cholesterol was performed by comparing the peak areas with the response from an internal standard. The percentage of cholesterol reduction was calculated as follows: cholesterol reduction $(\%)=100$ (amount of cholesterol in cream cheese). Cholesterol determinations for Gouda cheese were averaged with each batch of treatments. All experiments were performed in triplicate.

\section{Extraction of Flavor Compounds}

The edges of the frozen cheese samples were removed and samples were grated using a conventional grater. The flavor compounds from cheese sample were extracted by modified methods of Lee et al. (2003). The cheese samples were grated and $10 \mathrm{~g}$ of each cheese was placed in a $50-\mathrm{mL}$ vial containing $10 \mathrm{~mL}$ of $\mathrm{NaH}_{2} \mathrm{PO}_{4}$ (25\%, wt/vol) along with $7 \mathrm{~mL}$ of distilled water and $1 \mathrm{~mL}$ of internal standard [5-nonanon $(10 \mu \mathrm{g} / \mathrm{mL}$ in methanol); Delgado et al., 2010]. The vial was immediately sealed with an air-tight aluminum cap and rubber septum. The sample was magnetically stirred at 800 rpm at $50^{\circ} \mathrm{C}$ for $30 \mathrm{~min}$ to homogenize and accelerate equilibrium of head space volatile compounds between the cheese matrix and headspace. After $30 \mathrm{~min}$, the extraction of volatile compounds was performed by injecting the manual SPME holder unit equipped with a 50/30- $\mu \mathrm{m}$ divinylbenzene/Carboxen/polydimethylsiloxane SPME fiber (Supelco Inc.) into the vial and exposing it to the headspace at $50^{\circ} \mathrm{C}$ for $30 \mathrm{~min}$ with magnetic stirring $(500 \mathrm{rpm})$. The flavor compounds iso-
Table 1. Chemical composition of cholesterol-removed Gouda cheese (CRGC) and Gouda cheese (control)

\begin{tabular}{lcc}
\hline & \multicolumn{2}{c}{ Cheese type } \\
\cline { 2 - 3 } Item & CRGC & Control \\
\hline Moisture (\%) & $48.44^{\mathrm{a}}$ & $42.96^{\mathrm{b}}$ \\
Fat (\%) & $31.45^{\mathrm{a}}$ & $32.99^{\mathrm{a}}$ \\
Protein (\%) & $20.45^{\mathrm{a}}$ & $22.51^{\mathrm{a}}$ \\
Yield (\%) & $11.64^{\mathrm{a}}$ & $10.32^{\mathrm{a}}$ \\
Cholesterol (mg/100 g) & 7.83 & 82.41 \\
Cholesterol removal (\%) & 90.5 & - \\
\hline
\end{tabular}

$\overline{\mathrm{a}, \mathrm{b}}$ Means within the same row with the same letter are not significantly different $(P>0.05)$.

lated were directly adsorbed in the injection port of the gas chromatograph, which was set at $250^{\circ} \mathrm{C}$ for $2 \mathrm{~min}$ in splitless mode. Isolations of volatile flavor compounds were performed in duplicate.

\section{Identification of Flavor Compounds}

The identification of flavor compounds was performed using an Agilent 6890 gas spectrometer coupled to a 5793N mass selective detector (Agilent Technologies, Santa Clara, CA). The GC-MS transfer line was set at $230^{\circ} \mathrm{C}$. The mass spectrometer operated in electron impact mode with an electron impact energy of $70 \mathrm{eV}$ and collected data at a rate of $0.7 \mathrm{scan} / \mathrm{s}$ over a range of 40 to $650 \mathrm{~m} / z$. The eluted identification of flavor compounds was performed by comparing their retention times and mass spectra with those of standards and their mass spectra with the National Institute of Standards and Technology (NIST) mass spectral library (Gkatzionis et al., 2009). The retention indices of unknown compounds were determined via sample injection with a homologous series of n-alkanes (Sigma 68281; Kováts, 1965). The GC-MS conditions were the same as described above. The analyses of flavor compounds were performed on an Agilent 6890 gas spectrometer coupled to a $5793 \mathrm{~N}$ mass selective detector. Flavor compounds were separated using a DB-5 column $(30-\mathrm{m} \times 0.25-\mathrm{mm}$ i.d., $1.0-\mu \mathrm{m}$ film thickness; J\&W Scientific Inc., Folsom, CA). The carrier gas was helium with a flow of $1.3 \mathrm{~mL} / \mathrm{min}$. The temperature programmed was isothermal at $40^{\circ} \mathrm{C}$ for 5 min and then raised at $5^{\circ} \mathrm{C} / \mathrm{min}$ to $250^{\circ} \mathrm{C}$ and held for $10 \mathrm{~min}$.

\section{Quantification of Flavor Compounds}

To quantify the volatiles, the samples were run in duplicate using a gas chromatograph-FID, and the integrated areas based on the total ion chromatograms were normalized to the areas of the internal standard and averaged. The relative volatile concentrations in the samples were determined by comparison with the con- 
Table 2. Overlapping flavor compounds identified in cholesterol-removed Gouda cheese and control cheese ripened at $14^{\circ} \mathrm{C}$ for 6 mo

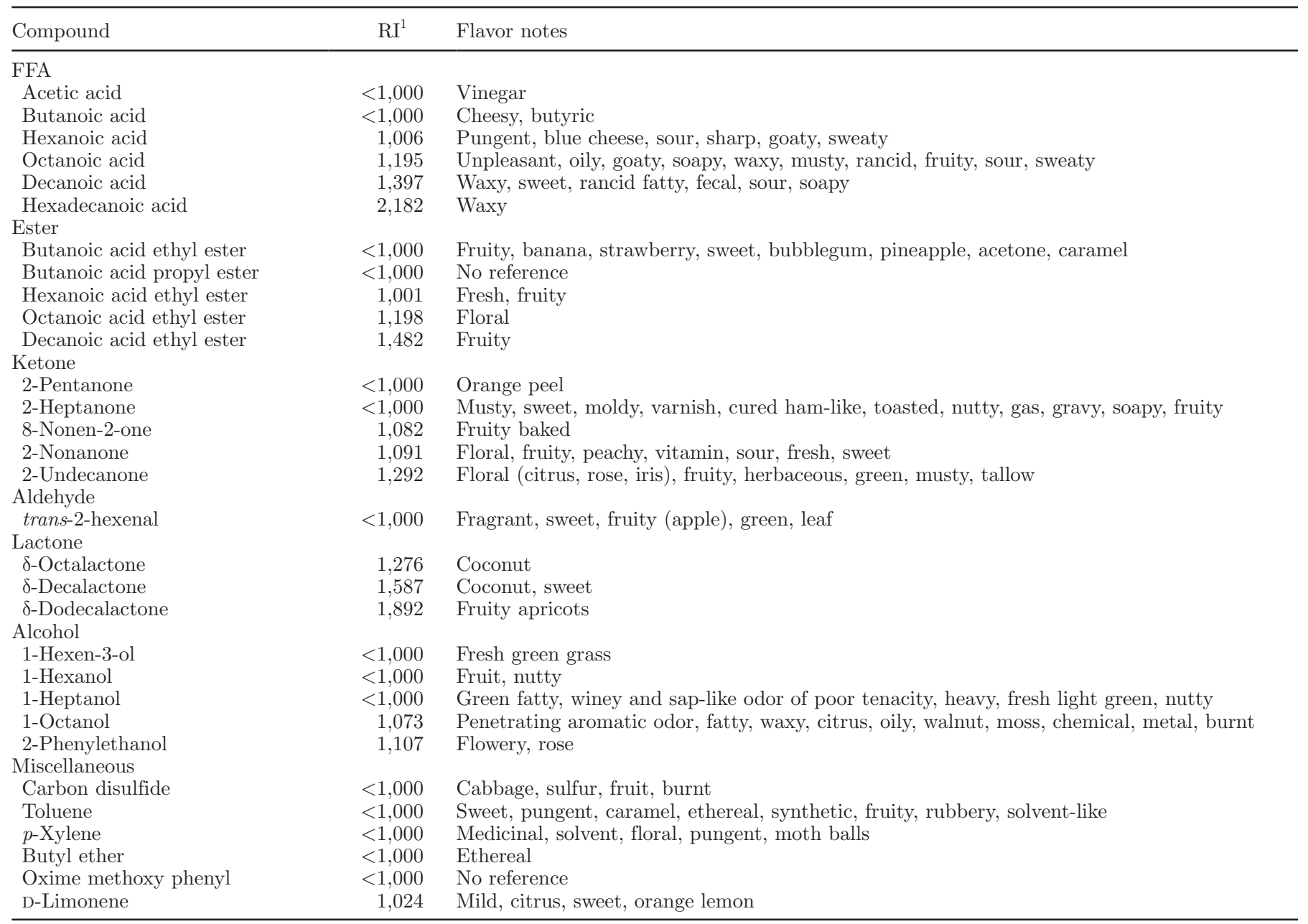

${ }^{1}$ Retention index.

centration of the internal standard (undecane), assuming a response factor of 1 . To determine the reproducibility of the duplicate injections and which peaks varied across samples, 2-way analyses of variance (sample and injection) were performed for each volatile peak.

\section{Analysis of Short-Chain FFA}

Ground Gouda cheese samples (1 g) were removed periodically from the cheeses after $0,1,2,3,4,5$, and $6 \mathrm{mo}$, extracted with $5 \mathrm{~mL}$ of diethyl ether, $2.5 \mathrm{~g}$ of

Table 3. Specific flavor compounds identified in cholesterol-removed Gouda cheese ripened at $14^{\circ} \mathrm{C}$ for 6 mo

\begin{tabular}{lrl}
\hline Compound & RI $^{1}$ & Flavor notes \\
\hline Ester & & \\
Acetic acid ethyl ester & $<1,000$ & Fruity, green \\
Dodecanoic acid ethyl ester & 1,695 & Waxy, fruity \\
$\begin{array}{c}\text { Aldehyde } \\
\text { Benzenealdehyde }\end{array}$ & $<1,000$ & Bitter almond, aromatic, fragrant, sweet, almond, nutty, fruity \\
Alcohol & $<1,000$ & No reference \\
2-Methyl-1-pentanol & $<1,000$ & Mushroom, earthy, musty \\
1-Octen-3-ol & $<1,000$ & Solvent odor \\
Miscellaneous & $<1,000$ & Toasted, nutty, roast grain \\
3-Methylhexane & $<1,000$ & Sharp, pine, green \\
2,6-Dimethylpyrazine & $<1,000$ & Sweet, balsamic, plastic \\
Q-Pinene & &
\end{tabular}


Table 4. Specific flavor compounds identified in control cheese ripened at $14^{\circ} \mathrm{C}$ for 6 mo

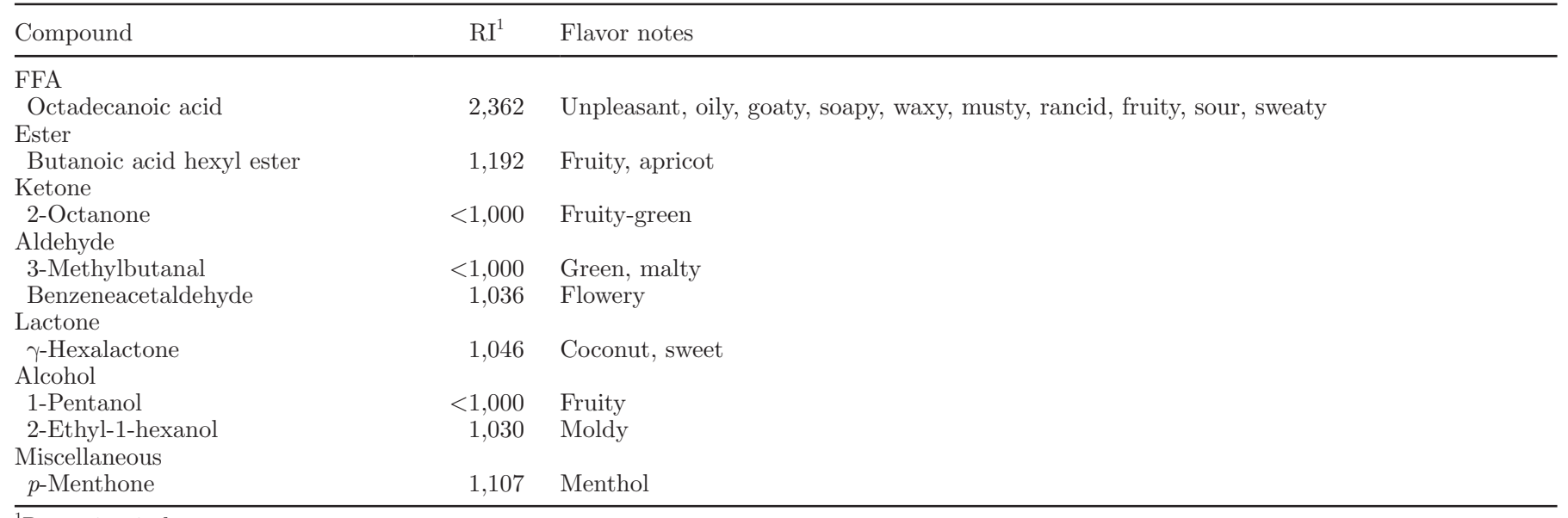

${ }^{1}$ Retention index.

Table 5. Concentration $(\mathrm{mg} / \mathrm{kg})$ of FFA and esters in cholesterol-removed Gouda cheese (CRGC) and Gouda cheese (control) ripened at $14^{\circ} \mathrm{C}$ for 6 mo

\begin{tabular}{|c|c|c|c|c|c|c|c|c|}
\hline \multirow[b]{2}{*}{ Compound } & \multirow{2}{*}{$\begin{array}{l}\text { Cheese } \\
\text { type }\end{array}$} & \multicolumn{7}{|c|}{ Ripening period (mo) } \\
\hline & & $0^{1}$ & 1 & 2 & 3 & 4 & 5 & 6 \\
\hline \multicolumn{9}{|l|}{ FFA } \\
\hline \multirow[t]{2}{*}{ Acetic acid } & CRGC & $13.78^{\mathrm{d}, \mathrm{A}}$ & $17.28^{\mathrm{cd}, \mathrm{A}}$ & $29.43^{\mathrm{a}, \mathrm{A}}$ & $25.29^{\mathrm{ab}, \mathrm{A}}$ & $21.29^{\mathrm{bc}, \mathrm{A}}$ & $21.35^{\mathrm{bc}, \mathrm{A}}$ & $21.54^{\mathrm{bc}, \mathrm{A}}$ \\
\hline & Control & $6.69^{\mathrm{a}, \mathrm{A}}$ & $18.52^{\mathrm{a}, \mathrm{A}}$ & $11.57^{\mathrm{a}, \mathrm{B}}$ & $27.20^{\mathrm{a}, \mathrm{A}}$ & $40.92^{\mathrm{a}, \mathrm{A}}$ & $27.83^{\mathrm{a}, \mathrm{A}}$ & $41.33^{\mathrm{a}, \mathrm{A}}$ \\
\hline \multirow[t]{2}{*}{ Butanoic acid } & $\mathrm{CRGC}$ & $1.42^{\mathrm{c}, \mathrm{A}}$ & $4.22^{\mathrm{bc}, \mathrm{A}}$ & $1.08^{\mathrm{c}, \mathrm{A}}$ & $6.49^{\mathrm{ab}, \mathrm{A}}$ & $8.21^{\mathrm{a}, \mathrm{A}}$ & $8.64^{\mathrm{a}, \mathrm{B}}$ & $8.03^{\mathrm{a}, \mathrm{A}}$ \\
\hline & Control & $0.81^{\mathrm{c}, \mathrm{A}}$ & $3.27^{\mathrm{bc}, \mathrm{A}}$ & $3.00^{\mathrm{bc}, \mathrm{A}}$ & $8.07^{\mathrm{abc}, \mathrm{A}}$ & $8.01^{\mathrm{abc}, \mathrm{A}}$ & $10.30^{\mathrm{ab}, \mathrm{A}}$ & $15.25^{\mathrm{a}, \mathrm{A}}$ \\
\hline \multirow[t]{2}{*}{ Hexanoic acid } & CRGC & $0.14^{\mathrm{b}, \mathrm{A}}$ & $4.73^{\mathrm{ab}, \mathrm{A}}$ & $1.51^{\mathrm{ab}, \mathrm{A}}$ & $0.24^{\mathrm{ab}, \mathrm{A}}$ & $2.40^{\mathrm{ab}, \mathrm{A}}$ & $5.66^{\mathrm{ab}, \mathrm{A}}$ & $6.81^{\mathrm{a}, \mathrm{A}}$ \\
\hline & Control & $0.74^{\mathrm{b}, \mathrm{A}}$ & $0.46^{\mathrm{b}, \mathrm{B}}$ & $0.53^{\mathrm{b}, \mathrm{A}}$ & $1.79^{\mathrm{ab}, \mathrm{A}}$ & $3.33^{\mathrm{ab}, \mathrm{A}}$ & $6.85^{\mathrm{a}, \mathrm{A}}$ & $7.57^{\mathrm{a}, \mathrm{A}}$ \\
\hline \multirow[t]{2}{*}{ Octanoic acid } & CRGC & $0.22^{\mathrm{a}, \mathrm{A}}$ & $0.35^{\mathrm{a}, \mathrm{A}}$ & $0.26^{\mathrm{a}, \mathrm{A}}$ & $0.39^{\mathrm{a}, \mathrm{A}}$ & $0.31^{\mathrm{a}, \mathrm{A}}$ & $0.24^{\mathrm{a}, \mathrm{A}}$ & $0.24^{\mathrm{a}, \mathrm{A}}$ \\
\hline & Control & $0.33^{\mathrm{a}, \mathrm{A}}$ & $0.52^{\mathrm{a}, \mathrm{A}}$ & $0.41^{\mathrm{a}, \mathrm{A}}$ & $0.57^{\mathrm{a}, \mathrm{A}}$ & $0.49^{\mathrm{a}, \mathrm{A}}$ & $0.62^{\mathrm{a}, \mathrm{A}}$ & $0.56^{\mathrm{a}, \mathrm{A}}$ \\
\hline \multirow[t]{2}{*}{ Decanoic acid } & CRGC & $0.21^{\mathrm{e}}$ & $0.71^{\mathrm{d}, \mathrm{A}}$ & $1.65^{\mathrm{c}, \mathrm{A}}$ & $0.96^{\mathrm{d}, \mathrm{A}}$ & $2.76^{\mathrm{b}, \mathrm{A}}$ & $2.85^{\mathrm{b}, \mathrm{A}}$ & $3.61^{\mathrm{a}, \mathrm{A}}$ \\
\hline & Control & $\mathrm{NQ}^{2}$ & $0.42^{\mathrm{b}, \mathrm{A}}$ & $0.50^{\mathrm{b}, \mathrm{B}}$ & $0.61^{\mathrm{b}, \mathrm{A}}$ & $1.53^{\mathrm{a}, \mathrm{B}}$ & $1.21^{\mathrm{a}, \mathrm{B}}$ & $1.15^{\mathrm{a}, \mathrm{B}}$ \\
\hline \multirow[t]{2}{*}{ Hexadecanoic acid } & CRGC & $0.65^{\mathrm{a}, \mathrm{A}}$ & $1.22^{\mathrm{a}, \mathrm{A}}$ & $1.88^{\mathrm{a}, \mathrm{A}}$ & $0.90^{\mathrm{a}, \mathrm{A}}$ & $1.48^{\mathrm{a}, \mathrm{A}}$ & $1.12^{\mathrm{a}, \mathrm{B}}$ & $0.67^{\mathrm{a}, \mathrm{A}}$ \\
\hline & Control & $0.91^{\mathrm{b}, \mathrm{A}}$ & $2.67^{\mathrm{a}, \mathrm{A}}$ & $0.72^{\mathrm{b}, \mathrm{A}}$ & $1.21^{\mathrm{ab}, \mathrm{A}}$ & $1.99^{\mathrm{ab}, \mathrm{A}}$ & $1.95^{\mathrm{ab}, \mathrm{A}}$ & $1.20^{\mathrm{ab}, \mathrm{A}}$ \\
\hline \multirow[t]{2}{*}{ Octadecanoic acid } & CRGC & $\mathrm{ND}^{3}$ & ND & ND & ND & ND & ND & ND \\
\hline & Control & $0.90^{\mathrm{a}}$ & $3.07^{\mathrm{a}}$ & NQ & $0.98^{\mathrm{a}}$ & $1.04^{\mathrm{a}}$ & $1.19^{\mathrm{a}}$ & $0.83^{\mathrm{a}}$ \\
\hline \multirow[t]{2}{*}{ Total } & CRGC & 16.42 & 28.51 & 35.81 & 34.27 & 36.45 & 39.86 & 40.90 \\
\hline & Control & 10.38 & 28.93 & 16.73 & 40.43 & 57.31 & 49.95 & 67.89 \\
\hline \multicolumn{9}{|l|}{ Ester } \\
\hline \multirow[t]{2}{*}{ Acetic acid-ethyl ester } & CRGC & $4.67^{\mathrm{a}}$ & $2.36^{\mathrm{bc}}$ & $2.08^{\mathrm{bc}}$ & $1.65^{\mathrm{c}}$ & $2.39^{\mathrm{bc}}$ & $1.92^{\mathrm{bc}}$ & $2.44^{\mathrm{b}}$ \\
\hline & Control & ND & ND & ND & ND & ND & ND & ND \\
\hline \multirow[t]{2}{*}{ Butanoic acid-ethyl ester } & CRGC & $6.49^{\mathrm{abc}, \mathrm{A}}$ & $5.37^{\mathrm{bc}, \mathrm{A}}$ & $6.75^{\mathrm{ab}, \mathrm{B}}$ & $7.41^{\mathrm{a}, \mathrm{A}}$ & $4.93^{\mathrm{bc}, \mathrm{B}}$ & $4.80^{\mathrm{c}, \mathrm{A}}$ & $4.75^{\mathrm{c}, \mathrm{B}}$ \\
\hline & Control & $8.39^{\mathrm{a}, \mathrm{A}}$ & $13.37^{\mathrm{a}, \mathrm{A}}$ & $14.78^{\mathrm{a}, \mathrm{A}}$ & $6.93^{\mathrm{a}, \mathrm{A}}$ & $15.06^{\mathrm{a}, \mathrm{A}}$ & $14.10^{\mathrm{a}, \mathrm{A}}$ & $10.63^{\mathrm{a}, \mathrm{A}}$ \\
\hline \multirow[t]{2}{*}{ Butanoic acid-propyl ester } & CRGC & $0.22^{\mathrm{c}}$ & $0.46^{\mathrm{bc}, \mathrm{A}}$ & $0.33^{\mathrm{c}, \mathrm{B}}$ & $0.38^{\mathrm{c}, \mathrm{B}}$ & $0.86^{\mathrm{a}, \mathrm{A}}$ & $0.64^{\mathrm{ab}, \mathrm{A}}$ & $0.87^{\mathrm{a}, \mathrm{A}}$ \\
\hline & Control & NQ & $0.54^{\mathrm{a}, \mathrm{A}}$ & $1.08^{\mathrm{a}, \mathrm{A}}$ & $0.89^{\mathrm{a}, \mathrm{A}}$ & $0.91^{\mathrm{a}, \mathrm{A}}$ & $1.27^{\mathrm{a}, \mathrm{A}}$ & $0.45^{\mathrm{a}, \mathrm{B}}$ \\
\hline \multirow[t]{2}{*}{ Hexanoic acid-ethyl ester } & CRGC & $1.41^{\mathrm{d}, \mathrm{A}}$ & $9.70^{\mathrm{b}, \mathrm{A}}$ & $5.17^{\mathrm{c}, \mathrm{A}}$ & $10.93^{\mathrm{ab}, \mathrm{A}}$ & $12.06^{\mathrm{a}, \mathrm{A}}$ & $12.22^{\mathrm{a}, \mathrm{A}}$ & $12.64^{\mathrm{a}, \mathrm{A}}$ \\
\hline & Control & $2.56^{\mathrm{b}, \mathrm{A}}$ & $3.56^{\mathrm{b}, \mathrm{B}}$ & $7.27^{\mathrm{b}, \mathrm{A}}$ & $2.95^{\mathrm{b}, \mathrm{B}}$ & $4.32^{\mathrm{b}, \mathrm{B}}$ & $5.61^{\mathrm{b}, \mathrm{A}}$ & $14.49^{\mathrm{a}, \mathrm{A}}$ \\
\hline \multirow[t]{2}{*}{ Butanoic acid-hexyl ester } & CRGC & ND & ND & ND & ND & ND & ND & ND \\
\hline & Control & NQ & NQ & $0.39^{\mathrm{b}}$ & $0.32^{\mathrm{b}}$ & $0.41^{\mathrm{b}}$ & $1.29^{\mathrm{a}}$ & $1.57^{\mathrm{a}}$ \\
\hline \multirow[t]{2}{*}{ Octanoic acid-ethyl ester } & CRGC & NQ & NQ & $0.14^{\mathrm{c}}$ & $0.38^{\mathrm{b}}$ & $0.48^{\mathrm{b}, \mathrm{A}}$ & $0.14^{\mathrm{c}, \mathrm{B}}$ & $1.37^{\mathrm{a}, \mathrm{A}}$ \\
\hline & Control & $\mathrm{NQ}$ & $\mathrm{NQ}$ & NQ & NQ & $0.33^{\mathrm{a}, \mathrm{A}}$ & $0.30^{\mathrm{a}, \mathrm{A}}$ & $0.32^{\mathrm{a}, \mathrm{B}}$ \\
\hline \multirow[t]{2}{*}{ Decanoic acid-ethyl ester } & CRGC & $\mathrm{NQ}$ & $\mathrm{NQ}$ & NQ & $0.57^{\mathrm{a}, \mathrm{A}}$ & $0.41^{\mathrm{b}, \mathrm{A}}$ & $0.56^{\mathrm{ab}, \mathrm{A}}$ & $0.18^{\mathrm{c}, \mathrm{A}}$ \\
\hline & Control & $\mathrm{NQ}$ & $\mathrm{NQ}$ & NQ & $0.44^{\mathrm{a}, \mathrm{A}}$ & $0.44^{\mathrm{a}, \mathrm{A}}$ & $0.60^{\mathrm{a}, \mathrm{A}}$ & $0.22^{\mathrm{a}, \mathrm{A}}$ \\
\hline \multirow[t]{2}{*}{ Dodecanoic acid-ethyl ester } & CRGC & $0.15^{\mathrm{a}}$ & $0.48^{\mathrm{a}}$ & $0.18^{\mathrm{a}}$ & $0.27^{\mathrm{a}}$ & $0.47^{\mathrm{a}}$ & $0.56^{\mathrm{a}}$ & $0.48^{\mathrm{a}}$ \\
\hline & Control & ND & ND & ND & ND & ND & ND & ND \\
\hline \multirow[t]{2}{*}{ Total } & CRGC & 12.94 & 18.37 & 14.65 & 21.59 & 21.60 & 20.84 & 22.73 \\
\hline & Control & 10.95 & 17.47 & 23.52 & 11.50 & 21.47 & 23.27 & 27.68 \\
\hline
\end{tabular}

${ }^{\mathrm{a}-\mathrm{e}}$ Means with different superscripts within a row are significantly different at $P<0.05$ by Duncan's multiple range test.

${ }^{\mathrm{A}, \mathrm{B}}$ Means with different superscripts within a column are significantly different at $P<0.05$ by Duncan's multiple range test.

${ }^{1}$ Sample was obtained $48 \mathrm{~h}$ after cheese making.

${ }^{2} \mathrm{NQ}=$ not quantified.

${ }^{3} \mathrm{ND}=$ not detected. 
Table 6. Concentration $(\mathrm{mg} / \mathrm{kg})$ of ketones and aldehydes in cholesterol-removed Gouda cheese (CRGC) and Gouda cheese (control) ripened at $14^{\circ} \mathrm{C}$ for $6 \mathrm{mo}$

\begin{tabular}{|c|c|c|c|c|c|c|c|c|}
\hline Compound & $\begin{array}{l}\text { Cheese } \\
\text { type }\end{array}$ & \multicolumn{7}{|c|}{ Ripening period (mo) } \\
\hline \multicolumn{9}{|l|}{ Ketone } \\
\hline 2-Pentanone & Control & $2.44^{\mathrm{b}, \mathrm{A}}$ & $7.11^{\mathrm{a}, \mathrm{A}}$ & $7.03^{\mathrm{a}, \mathrm{A}}$ & $3.02^{\mathrm{b}, \mathrm{A}}$ & $2.42^{\mathrm{b}, \mathrm{A}}$ & $7.01^{\mathrm{a}, \mathrm{A}}$ & $5.17^{\mathrm{a}, \mathrm{A}}$ \\
\hline \multirow[t]{2}{*}{ 2-Heptanone } & CRGC & $0.85^{\mathrm{d}, \mathrm{A}}$ & $1.62^{\mathrm{d}, \mathrm{B}}$ & $0.84^{\mathrm{d}, \mathrm{B}}$ & $3.67^{\mathrm{c}, \mathrm{A}}$ & $5.08^{\mathrm{b}, \mathrm{B}}$ & $6.10^{\mathrm{a}, \mathrm{A}}$ & $6.41^{\mathrm{a}, \mathrm{B}}$ \\
\hline & Control & $2.08^{\mathrm{c}, \mathrm{A}}$ & $4.37^{\mathrm{c}, \mathrm{A}}$ & $6.74^{\mathrm{c}, \mathrm{A}}$ & $6.24^{\mathrm{c}, \mathrm{A}}$ & $19.37^{\mathrm{b}, \mathrm{A}}$ & $26.02^{\mathrm{ab}, \mathrm{A}}$ & $31.91^{\mathrm{a}, \mathrm{A}}$ \\
\hline \multirow[t]{2}{*}{ 8-Nonen-2-one } & CRGC & $\mathrm{NQ}^{3}$ & $0.46^{\mathrm{bc}, \mathrm{A}}$ & $\mathrm{NQ}$ & $0.23^{\mathrm{c}, \mathrm{B}}$ & $0.77^{\mathrm{ab}, \mathrm{A}}$ & $0.62^{\mathrm{ab}, \mathrm{A}}$ & $0.84^{\mathrm{a}, \mathrm{A}}$ \\
\hline & Control & $0.29^{\mathrm{e}}$ & $0.48^{\text {cde }, \mathrm{A}}$ & $0.38^{\mathrm{de}}$ & $0.60^{\mathrm{bcd}, \mathrm{A}}$ & $0.73^{\mathrm{abc}, \mathrm{A}}$ & $0.75^{\mathrm{ab}, \mathrm{A}}$ & $0.90^{\mathrm{a}, \mathrm{A}}$ \\
\hline \multirow[t]{2}{*}{ 2-Nonanone } & CRGC & NQ & $0.81^{\mathrm{bc}, \mathrm{A}}$ & $0.34^{\mathrm{c}, \mathrm{B}}$ & $0.38^{\mathrm{c}, \mathrm{A}}$ & $1.25^{\mathrm{ab}, \mathrm{B}}$ & $1.18^{\mathrm{ab}, \mathrm{A}}$ & $1.68^{\mathrm{a}, \mathrm{B}}$ \\
\hline & Control & $0.32^{\mathrm{c}}$ & $0.75^{\mathrm{c}, \mathrm{A}}$ & $1.08^{\mathrm{c}, \mathrm{A}}$ & $1.46^{\mathrm{c}, \mathrm{A}}$ & $6.42^{\mathrm{b}, \mathrm{A}}$ & $9.58^{\mathrm{a}, \mathrm{A}}$ & $8.92^{\mathrm{a}, \mathrm{A}}$ \\
\hline \multirow{2}{*}{ 2-Undecanone } & CRGC & $0.13^{\mathrm{b}, \mathrm{A}}$ & $0.20^{\mathrm{a}, \mathrm{B}}$ & $0.20^{\mathrm{a}, \mathrm{A}}$ & $0.19^{\mathrm{a}, \mathrm{A}}$ & $0.21^{\mathrm{a}, \mathrm{A}}$ & $0.20^{\mathrm{a}, \mathrm{A}}$ & $0.19^{\mathrm{a}, \mathrm{A}}$ \\
\hline & Control & $0.21^{\mathrm{ab}, \mathrm{A}}$ & $0.36^{\mathrm{ab}, \mathrm{A}}$ & $0.37^{\mathrm{a}, \mathrm{A}}$ & $0.17^{\mathrm{b}, \mathrm{A}}$ & $0.35^{\mathrm{ab}, \mathrm{A}}$ & $0.32^{\mathrm{ab}, \mathrm{A}}$ & $0.24^{\mathrm{ab}, \mathrm{A}}$ \\
\hline Total & CRGC & 1.96 & 3.89 & 2.58 & 5.45 & 8.87 & 9.69 & 11.32 \\
\hline trans-2-hexenal & Control & NQ & NQ & $2.14^{\mathrm{d}, \mathrm{A}}$ & $3.44^{\mathrm{cd}, \mathrm{A}}$ & $3.56^{\mathrm{c}, \mathrm{A}}$ & $6.45^{\mathrm{b}, \mathrm{A}}$ & $8.47^{\mathrm{a}, \mathrm{A}}$ \\
\hline \multirow[t]{2}{*}{ Benzaldehyde } & CRGC & ND & ND & ND & ND & ND & ND & ND \\
\hline & Control & ND & ND & ND & ND & ND & ND & ND \\
\hline \multirow[t]{2}{*}{ Benzeneacetaldehyde } & CRGC & ND & ND & ND & ND & ND & ND & ND \\
\hline & Control & NQ & NQ & $0.41^{\mathrm{cd}}$ & $0.35^{\mathrm{d}}$ & $0.54^{\mathrm{bc}}$ & $0.63^{\mathrm{a}}$ & $0.59^{\mathrm{ab}}$ \\
\hline \multirow[t]{2}{*}{ Total } & CRGC & 0.72 & 1.70 & 2.55 & 4.82 & 3.89 & 5.01 & 4.63 \\
\hline & Control & 1.42 & 2.06 & 4.80 & 6.30 & 5.30 & 10.41 & 14.51 \\
\hline
\end{tabular}

${ }^{\mathrm{a}}{ }^{\mathrm{e}}$ Means with different superscripts within a row are significantly different at $P<0.05$.

${ }^{\mathrm{A}, \mathrm{B}}$ Means with different superscripts within a column are significantly different at $P<0.05$.

${ }^{1}$ Sample was obtained $48 \mathrm{~h}$ after cheese making.

${ }^{2} \mathrm{ND}=$ not detected.

${ }^{3} \mathrm{NQ}=$ not quantified.

sodium sulfate, and $0.1 \mathrm{~mL}$ of $4 \mathrm{~N} \mathrm{H}_{2} \mathrm{SO}_{4}$ for $2 \mathrm{~h}$ and eluted through a 10-mm-i.d. glass column containing 1 $\mathrm{g}$ of deactivated alumina (alumina oxide neutral was deactivated with $0.24 \%$ distilled water, as described by Kwak et al. (1990). An analysis of short-chain FFA was performed using Donam Instruments Inc. DS 6200 gas chromatograph. The preparation of FFA was achieved using a $15-\mathrm{m} \times 0.53-\mathrm{mm}-$ i.d. Nukol fused silica capillary column (Supelco Inc.). The gas chromatograph was operated with nitrogen carrier gas at $30 \mathrm{~mL} / \mathrm{min}$, hydrogen gas at $30 \mathrm{~mL} / \mathrm{min}$, and air at $300 \mathrm{~mL} / \mathrm{min}$. The column oven was programmed for initial holding for $1 \mathrm{~min}$ at $60^{\circ} \mathrm{C}$ and then heating to $170^{\circ} \mathrm{C}$ at $5^{\circ} \mathrm{C} /$ min for $1 \mathrm{~min}$. The temperature for both the injector and the detector was $220^{\circ} \mathrm{C}$. All experiments were performed in triplicate. Each peak was quantified by the retention time of reference standards.

\section{Statistical Analysis}

All statistical analyses were performed using SAS version 9.0 (SAS Institute, 1992). An ANOVA was performed using the general linear models procedure to de- termine significant differences among the samples. For pattern recognition, the principal component analysis (PCA) technique (Manly, 1986) was applied. Means were compared using Duncan's multiple range test $(P$ $<0.05$ ) and XLSTAT ver.2011 (Addinsoft Inc., New York, NY) was used for PCA.

\section{RESULTS AND DISCUSSION}

\section{Chemical Composition}

The chemical composition, cheese yield, and cholesterol contents of the CRGC and control cheese are shown in Table 1. The fat and protein contents had no significant difference between the CRGC and control $(P>0.05)$. However, the moisture content in CRGC was higher than that of control cheese. Similarly, higher moisture content was observed in certain low-fat cheeses, such as reduced-fat Cheddar cheese (Metzger and Mistry, 1994) due to the lower curd drainage. Further cholesterol removal was about $90.5 \%$, which was quite similar to that of cream cheese (Kim et al., 2005). 
Table 7. Concentration $(\mathrm{mg} / \mathrm{kg})$ of lactones and alcohols in cholesterol-removed Gouda cheese (CRGC) and Gouda cheese (control) ripened at $14^{\circ} \mathrm{C}$ for $6 \mathrm{mo}$

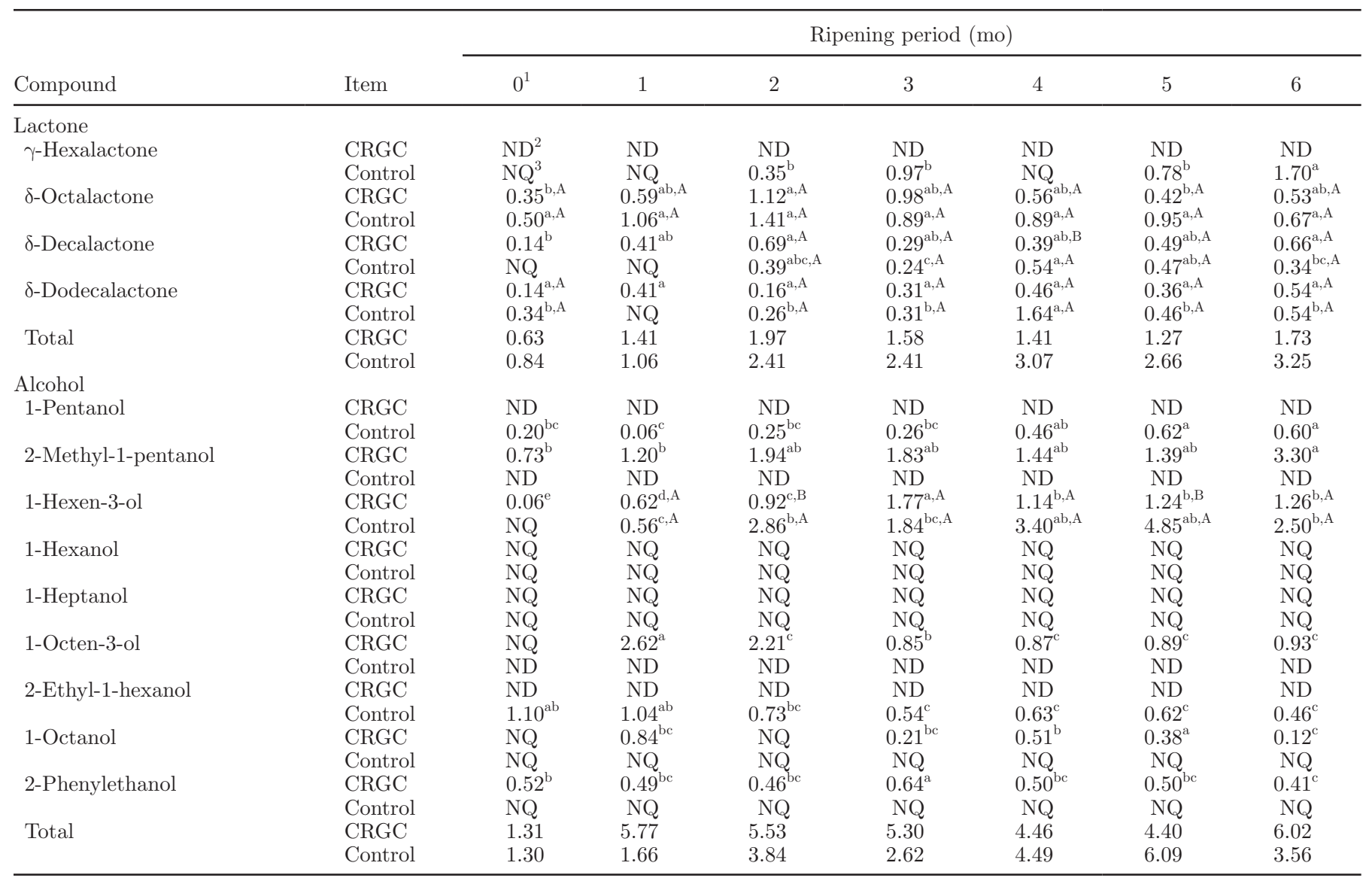

${ }^{\mathrm{a}-\mathrm{e}}$ Means with different superscripts within a row are significantly different at $P<0.05$.

${ }^{\mathrm{A}, \mathrm{B}}$ Means with different superscripts within a column are significantly different at $P<0.05$.

${ }^{1}$ Sample was obtained $48 \mathrm{~h}$ after cheese making.

${ }^{2} \mathrm{ND}=$ not detected.

${ }^{3} \mathrm{NQ}=$ not quantified.

\section{Identification of Flavor Compounds}

Flavor compounds isolated from control cheese and CRGC by SPME-GC-MS during 6 mo of ripening are shown in Tables 2, 3, and 4, and were related to their origin and to their sensory characteristics. A total of 40 flavor compounds were isolated in both cheeses and some differences were observed between samples. In both cheeses, 31 overlapping flavor compounds were detected, including 6 FFA, 5 esters, 5 ketones, 1 aldehyde, 3 lactones, 5 alcohols, and 6 miscellaneous compounds that could not be classified in these groups.

In addition to overlapping flavor compounds, CRGC showed 9 specific flavor compounds, including 2 esters, 1 aldehyde, 2 alcohols, and 4 miscellaneous compounds (Table 3). Similarly control cheese also showed a total of 9 flavor compounds that included 1 FFA, 1 ester, 1 ketone, 2 aldehydes, 1 lactone, 2 alcohols, and 1 miscel- laneous compound (Table 4). Similar flavor compounds were also observed in other Gouda-type cheeses and hard-type cheeses such as Appenzeller, Emmental, and Raclette cheeses (Lawlor et al., 2002). Free fatty acids, lactones, and ethyl esters are fat-derived flavor compounds that play an important role in the overall flavor of Gouda cheeses (Alewijin et al., 2005) due to their low aroma thresholds. 2-Methyketones $\left(\mathrm{C}_{5}\right.$ to $\left.\mathrm{C}_{15}\right)$ with odd carbon number are most abundant in most dairy products and they have typical odor characteristics and low perception thresholds. These compounds are formed by $\beta$-oxidation and decarboxylation of FA. It is known that aldehydes are not the major compounds in cheeses, as they are rapidly converted to alcohols or their corresponding acids (Lemieux and Simard, 1992). Branched-chain aldehydes (3-methylbutanal) are formed by the catabolism of branched-chain amino acids by an aminotransferase (Atiles et al., 2000; Marilley and 
Table 8. Concentration ( $\mathrm{mg} / \mathrm{kg}$ ) of miscellaneous compounds in cholesterol-removed Gouda cheese (CRGC) and Gouda cheese (control) ripened at $14^{\circ} \mathrm{C}$ for $6 \mathrm{mo}$

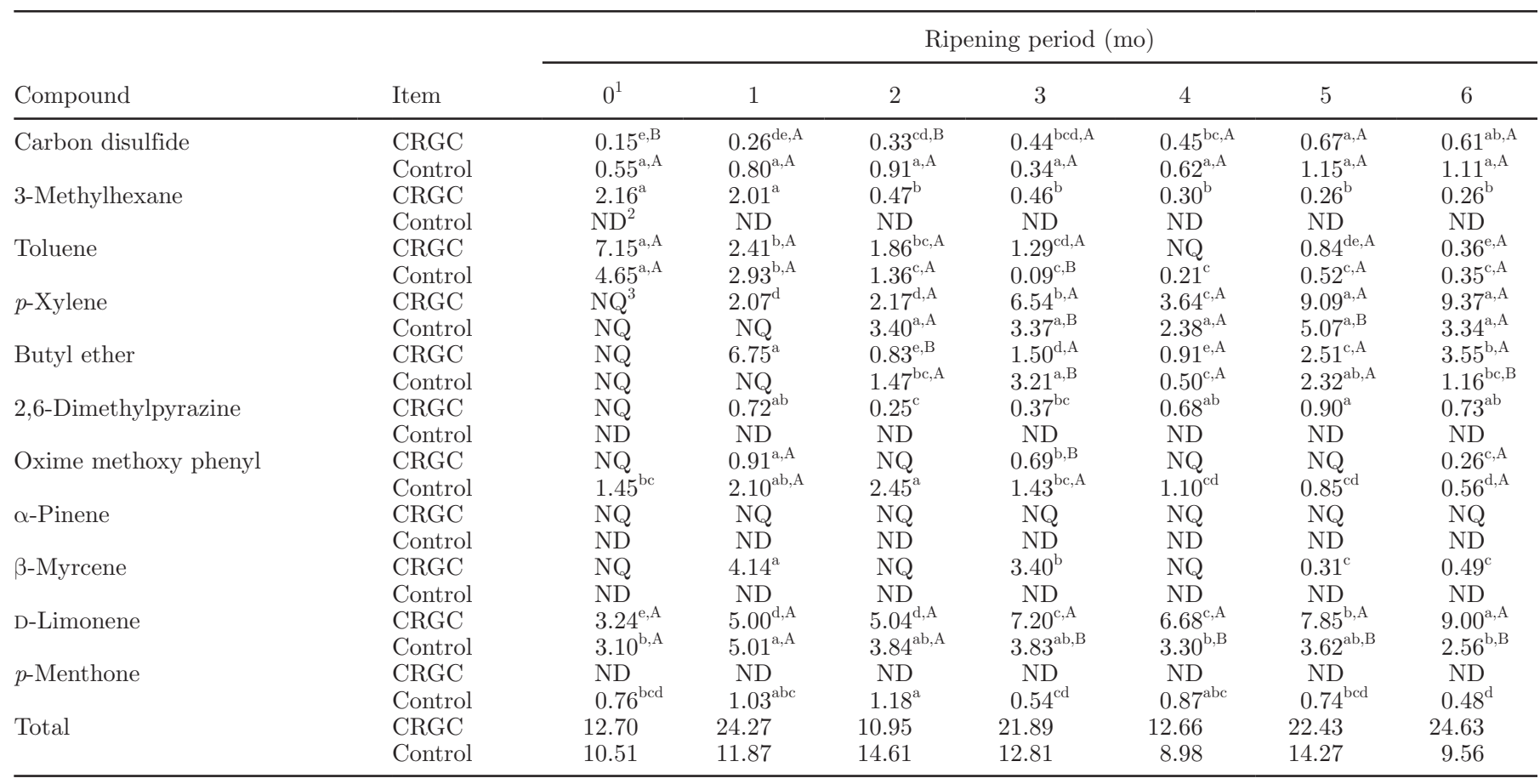

${ }^{\mathrm{a} e}$ Means with different superscripts within a row are significantly different at $P<0.05$.

${ }^{\mathrm{A}, \mathrm{B}}$ Means with different superscripts within a column are significantly different at $P<0.05$.

${ }^{1}$ Sample was obtained $48 \mathrm{~h}$ after cheese making.

${ }^{2} \mathrm{ND}=$ not detected.

${ }^{3} \mathrm{NQ}=$ not quantified.

Casey, 2004). Compounds such as benzaldehyde and 2-phenylethanol were formed by catabolism of aromatic amino acids (McSweeney and Sousa, 2000; Marilley et al., 2004). The low-threshold sulfur compounds toluene and $p$-xylene were seen in both cheeses, but they did not contribute much to cheese aroma (Frank et al., 2004). D-Limonene cannot be classified into a chemical class and, according to Mallia et al. (2005), it is identified in European Protected Designation of Origin (PDO) hard cheeses, such as Gruyère, Manchego, and Ragusano.

\section{Quantification of Flavor Compounds}

The flavor compounds $(\mathrm{mg} / \mathrm{kg}$ ) from CRGC and control cheese by SPME-GC-FID during 6 mo of ripening are shown in Tables 5, 6, 7, and 8. An increase in the ripening time significantly increased the flavor compound formation in both cheeses $(P<0.05)$. Free fatty acid was the most abundant flavor compound quantified in CRGC and control cheese during 6 mo of ripening (Table 5), which may serve as an important contributor to the flavor profile of various cheeses (Delgado et al., 2010). In the early stage of ripening, the concentration of flavor compounds quantified in
CRGC and control cheese were 16.42 and $10.38 \mathrm{mg} /$ $\mathrm{kg}$, respectively. At $6 \mathrm{mo}$, they increased to 40.90 and $67.89 \mathrm{mg} / \mathrm{kg}$, respectively. Among FFA, significant productions of acetic acid and butanoic acid were noticed $(P<0.05)$. In the case of acetic acid, it originated from different reactions by the lactic acid bacteria (Ur-Rehman et al., 2000) and its production was already abundant compared with other FFA at the early stage of ripening and was increased until the end of ripening; however, no significant difference was found $(P>0.05)$. Butanoic acid was the second abundant flavor compound quantified in both cheeses and increased significantly during the ripening $(P<0.05)$. This result was also related with cheesy odor, which could be associated with the cheesy flavor of Gouda cheese. Additionally, evolution of hexanoic acid, decanoic acid, and hexadecanoic acid was found. However, no significant evolution was found in octanoic acid, hexadecanoic acid, and octadecanoic acid $(P>0.05)$. In the results of chemical composition, the fat contents were 31.45 (CRGC) and $32.99 \%$ (control cheese). A small loss of fat (1.5\%) may lead to certain losses in flavor compounds. However, sensory evaluation was not conducted and follow-up studies are necessary to 


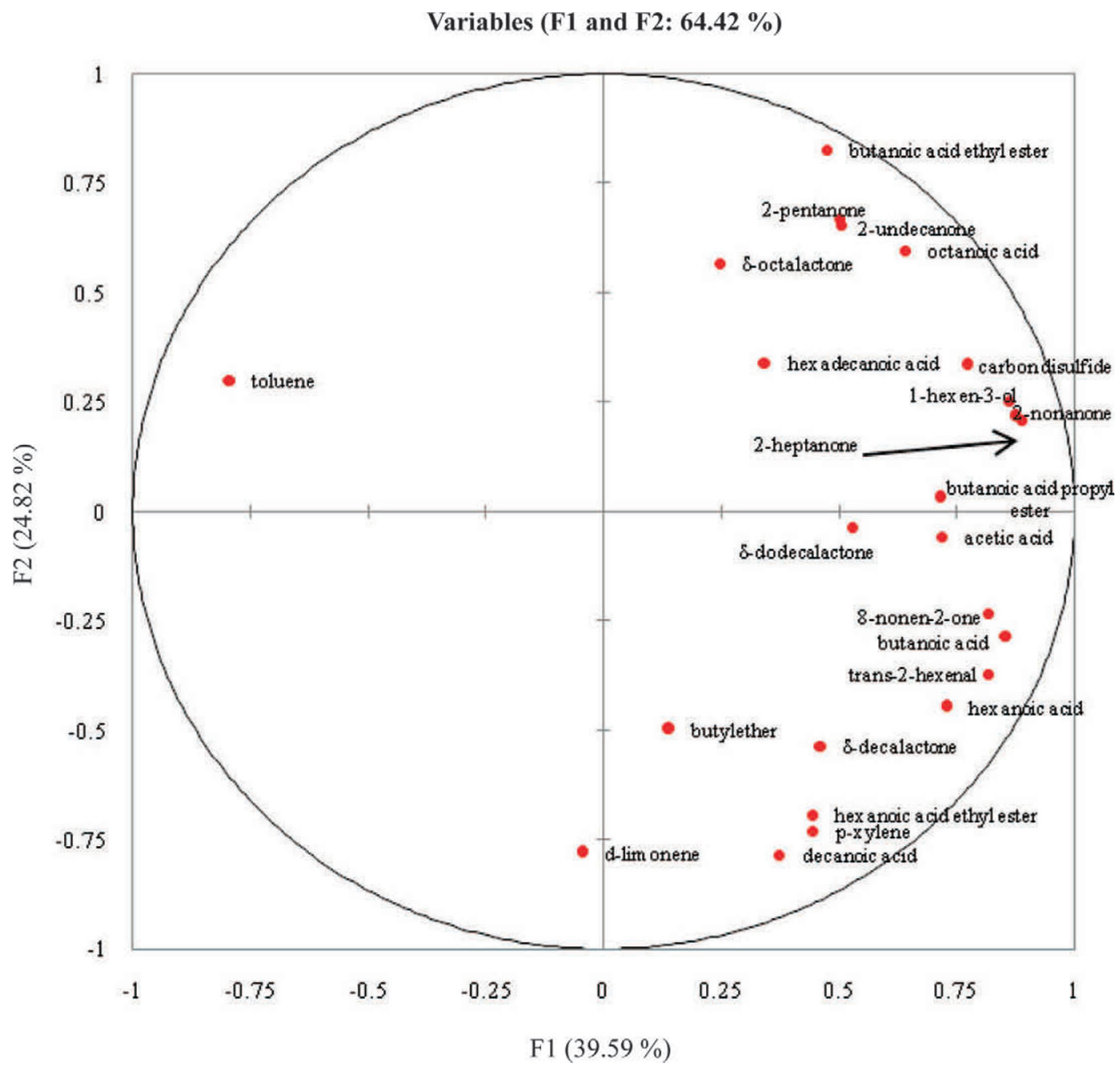

Figure 1. Principal component analysis (PCA) loadings for 24 flavor compounds. F1 = principal component 1; F2 = principal component 2. Arrow indicates position of 2-heptanone. Color version available in the online PDF.

confirm that the changes observed chemically translate to sensory quality changes.

Ripening increased ester flavor formation in CRGC and control (Table 5). At the early stage of ripening, total concentrations of esters were 12.94 (CRGC) and $10.95 \mathrm{mg} / \mathrm{kg}$ (control cheese) and they increased to 22.73 (CRGC) and $27.68 \mathrm{mg} / \mathrm{kg}$ (control cheese) at the end of ripening. Butanoic acid ethyl ester and hexanoic acid ethyl ester were the main contributors to evolution of ethyl esters during ripening. Butanoic acid hexyl ester increased significantly in the control cheese during the 6 mo of storage $(P<0.05)$. Octanoic acid ethyl ester was newly formed at the middle stage of ripening and increased during ripening. Decanoic acid ethyl esters formed at 3 mo of ripening; however, their production was stable. Dodecanoic acid ethyl ester was only isolated in CRGC.
Increases in the ripening period also favored ketone formation in CRGC and control cheese (Table 6). At the beginning of ripening, the total concentrations of ketones were 1.96 (CRGC) and $6.49 \mathrm{mg} / \mathrm{kg}$ (control cheese). After 6 mo of ripening, total concentrations reached 11.32 (CRGC) and $52.43 \mathrm{mg} / \mathrm{kg}$ (control cheese). However, the rate evolution of flavor compounds was different. 2-Heptanone and 2-nonanone were the most abundant ketones during ripening and their production was significantly increased $(P<0.05)$ as ripening progressed. Also the levels of 2-pentanone and 8-nonen-2-one and 2-octanone were significantly increased as ripening progressed $(P<0.05)$. In this study, aldehydes were not the main flavor compounds in Gouda cheese (Table 6); even their total production was relatively low. It was reported that the low level of aldehydes indicated optimal maturation because 
Observations (F1 and F2: $64.42 \%$ )

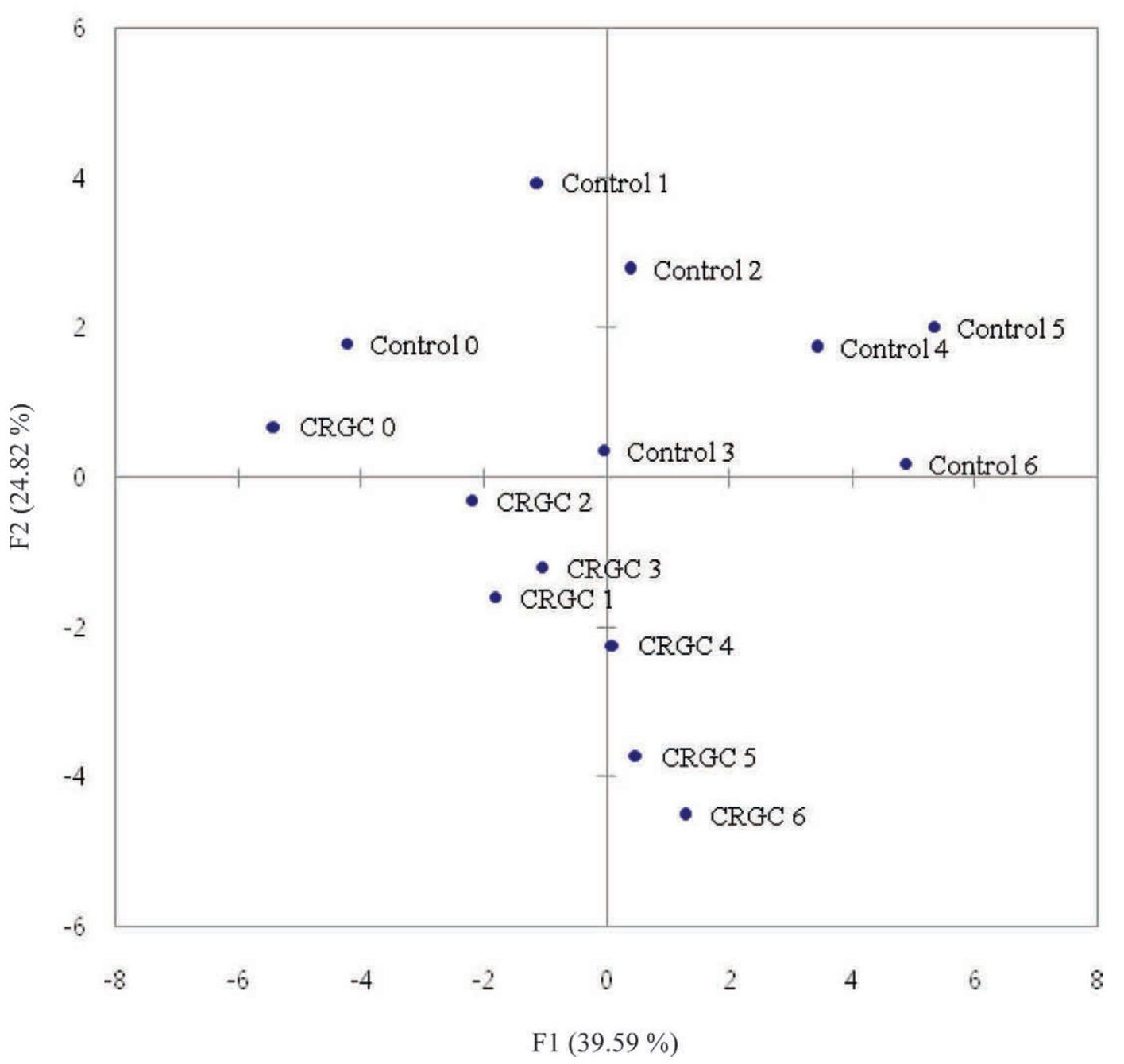

Figure 2. Principal component analysis (PCA) loadings for 14 cheese samples including cholesterol-removed Gouda cheeses (CRGC) and control ripened at $14^{\circ} \mathrm{C}$ for 6 mo. $\mathrm{F} 1=$ principal component $1 ; \mathrm{F} 2=$ principal component 2 . Color version available in the online $\mathrm{PDF}$.

a high concentration of aldehydes may result in offflavors (Moio and Addeo, 1998). Ripening increased the aldehyde concentration in CRGC and control cheese, from 0.72 and $1.42 \mathrm{mg} / \mathrm{kg}$ to 4.63 and $14.51 \mathrm{mg} / \mathrm{kg}$, respectively. The difference in total concentration between control cheese and CRGC was derived from the evolution of 3-methylbutanal (only control cheese) and trans-2-hexenal (both). Benzeneacetaldehyde was uniquely detected in control cheese; it was newly formed at 3 mo and tended to increase during ripening. In the case of lactones, at the early stage of ripening, total concentrations of CRGC and control cheese were 0.63 and $0.84 \mathrm{mg} / \mathrm{kg}$, respectively, and then increased to 1.73 (CRGC) and $3.25 \mathrm{mg} / \mathrm{kg}$ (control cheese) at the end of ripening (Table 7 ). However, most of the lactones showed no significant differences during ripening $(P>0.05)$.

In the case of alcohols, even though they were identified in GC-MS, most of them were not quantified in the gas chromatograph-FID and their levels were relatively low compared with other flavor compounds (Table 7). Initial total productions in CRGC and control cheese were 1.31 and $1.30 \mathrm{mg} / \mathrm{kg}$, respectively, and reached 6.02 (CRGC) and $3.56 \mathrm{mg} / \mathrm{kg}$ (control cheese) at the end of ripening. 1-Pentanol was only detected in control cheese and increased significantly $(P<0.05)$. 2-Methyl1-pentanol was only detected in CRGC and increased significantly $(P<0.05)$. The 1-hexen-3-ol detected in both cheeses tended to increase during ripening. Certain flavor alcohol compounds such as 1-octen-3-ol 
(CRGC only), 2-phenylethanol (CRGC only), and 2-ethyl-1-hexanol (control cheese only) were found to decrease during the ripening period. It was reported that these different patterns could be associated with the different metabolic pathways involved in the formation of alcohols in cheese, namely lactose metabolism, methyl ketone reduction, amino acid metabolism, and degradation of linoleic acid and linolenic acids (Molimard and Spinnler, 1996).

Regarding miscellaneous compounds, the results are shown in Table 8. As the only sulfur compound, carbon disulfide was detected from both cheeses and increased slightly during ripening. As a branched alkane, 3-methylhexane was quantified only in CRGC and its level was decreased significantly $(P<0.05)$ from 2.16 to $0.26 \mathrm{mg} /$ $\mathrm{kg}$ during ripening. Toluene showed a similar pattern and its level tended to decrease. The 4 terpenes, including $p$-xylene, $\alpha$-pinene, $\beta$-myrcene, and D-limonene showed different patterns during ripening. $\beta$-Myrcene was detected only in CRGC and significantly decreased during ripening $(P<0.05)$. $p$-Menthone was only identified in control cheese and showed no significant difference during ripening $(P>0.05)$. The level of Dlimonene tended to increase in CRGC; however, it was stable in control cheese.

The PCA plots of 24 flavor compounds and 14 cheese samples are illustrated in Figures 1 and 2. The plot explained $64.42 \%$ [principal component $1(\mathbf{F 1})=$ $39.59 \%$; principal component $2(\mathbf{F 2})=24.82 \%$ ] of the total variance. Major flavor compounds were noticed in the positive F1 and F2 axes of the PCA (Figure 1). Ripening caused a shift from the negative F1 axis to the positive $\mathrm{F} 1$ axis in both cheeses for various months; however, half of CRGC was seen in the negative F2 axis (Figure 2).

\section{CONCLUSIONS}

The results of this study confirmed that cholesterol removal was about $90 \%$ in Gouda cheese. The increase in the ripening period of Gouda cheese to 6 mo greatly increased the measurable flavor compounds in both cheeses. Among the flavor compounds generated, FFA and esters contributed higher amounts in CRGC at the initial stage and decreased during storage compared with the control cheese. Thus, this study confirmed that cholesterol removal greatly influences the flavor biochemistry of Gouda cheese. Sensory evaluation has yet to confirm practical implications of the findings.

\section{ACKNOWLEDGMENTS}

This study was supported by the Ministry for Food, Agriculture, Forestry and Fisheries Project in Seoul, Republic of Korea.

\section{REFERENCES}

Adams, M. L., D. M. Sullivan, R. L. Smith, and E. F. Richter. 1986. Evaluation of direct saponification method for determination of cholesterol in meats. J. Assoc. Off. Anal. Chem. 69:844-846.

Alewijin, M., E. L. Sliwinski, and J. T. M. Wouters. 2005. Production of fat-derived (flavour) compounds during the ripening of Gouda cheese. Int. Dairy J. 15:733-740.

AOAC International. 2000. Official Methods of Analysis. Vol. I. 19th ed. AOAC International, Washington, DC.

Atiles, M. W., E. G. Dudley, and J. L. Steele. 2000. Gene cloning, sequencing and inactivation of the branched-chain aminotransferase of Lactococcus lactis LM0230. Appl. Environ. Microbiol. 66:2325-2329.

BSI (British Standards Institution). 1969. Gerber method for the determination of fat in milk and milk products. Part 2. Methods. British Standard 696-2. British Standards Institution, London, UK.

Chamba, J. F., and É. Perreard. 2002. Contribution of propionic acid bacteria to lipolysis of Emmental cheese. Lait 82:33-44.

Delgado, F. J., J. González-Crespo, R. Cava, J. García-Parra, and R. Ramírez. 2010. Characterisation by SPME-GC-MS of the volatile profile of a Spanish soft cheese P.D.O. Torta del Casar during ripening. Food Chem. 118:182-189.

Frank, D. C., C. M. Owen, and J. Patterson. 2004. Solid phase microextraction (SPME) combined with gas-chromatography and olfactometry-mass spectrometry for characterization of cheese aroma compounds. Lebenson. Wiss. Technol. 37:139-154.

Gkatzionis, K., R. S. T. Linforth, and C. E. R. Dodd. 2009. Volatile profile of Stilton cheeses: Differences between zones within a cheese and dairies. Food Chem. 13:506-512.

Han, E. M., S.-H. Kim, J. Ahn, and H.-S. Kwak. 2007. Optimizing cholesterol removal from cream using $\beta$-cyclodextrin cross-linked with adipic acid. Int. Dairy Technol. 60:31-36.

Jeon, S. S., P. Ganesan, Y. S. Lee, S. H. Yoo, and H. S. Kwak. 2012a. Texture and sensory properties of cream cheese and cholesterolremoved cream cheese made from whole milk powder. Kor. J. Food Sci. Anim. Resour. 32:49-53.

Jeon, S. S., S. J. Lee, P. Ganesan, and H. S. Kwak. 2012b. Comparative study of flavor, texture, and sensory in cream cheese and cholesterol-removed cream cheese. Food Sci. Biotechnol. 21:159-165.

Kim, S. H., E. M. Han, J. Ahn, and H. S. Kwak. 2005. Effects of crosslinked $\beta$-cyclodextrin on quality of cholesterol-reduced cream cheese. Asian-australas. J. Anim. Sci. 18:584-589.

Kinsella, J. E. 1975. Butter flavor. Food Technol. 29:82, 84, 86, 88, 90, 92, 96, 98

Kosikowski, F. V., and V. V. Mistry. 1997. Cheese and Fermented Milk Foods, Volume 2: Procedures and Analysis. 3rd ed. F. V. Kosikowski LLC, Great Falls, VA.

Kováts, E. 1965. Gas chromatographic characterization of organic substances in the retention index system. Adv. Chromatogr. 1:229247.

Kwak, H. S., I. J. Jeon, and J. Park. 1990. Effects of food grade porcine pancreatic lipase on the production of short-chain fatty acids and its constitution. Kor. J. Food Sci. and Technol. 22:248-254.

Lawlor, J. B., C. M. Delahunty, M. G. Wilkinson, and J. Sheehan 2002. Relationships between the gross, non-volatile and volatile compositions and the sensory attributes of eight hard-type cheeses. Int. Dairy J. 12:493-509.

Lee, J. H., R. Diono, G. Y. Kim, and D. B. Min. 2003. Optimization of solid phase microextraction analysis for the headspace volatile compounds of parmesan cheese. J. Agric. Food Chem. 51:11361140

Lemieux, L., and R. E. Simard. 1992. Bitter flavour in dairy products. II. A review of bitter peptides from caseins: Their formation, isolation and identification, structure masking and inhibition. Lait 72:335-382.

Mallatou, H., E. Pappa, and T. Massouras. 2003. Changes in free fatty acids during ripening of Teleme cheese made with ewes', goats', cows' or a mixture of ewes' and goats' milk. Int. Dairy J. $13: 211-219$ 
Mallia, S., E. Fernández-García, and J. Olivier Bosset. 2005. Comparison of purge and trap and solid phase microextraction techniques for studying the volatile aroma compounds of three European PDO hard cheese. Int. Dairy J. 15:741-758.

Manly, B. J. F. 1986. Multivariate Methods. Chapman \& Hall, London, UK.

Marilley, L., and M. G. Casey. 2004. Flavours of cheese products: Metabolic pathways, analytical tools and identification of producing strains. Int. J. Food Microbiol. 90:139-159.

McSweeney, P. L. H., and M. J. Sousa. 2000. Biochemical pathways for the production of flavour compounds in cheese during ripening: A review. Lait 80:293-324.

Metzger, L. E., and V. V. Mistry. 1994. A new approach using homogenization of cream in the manufacture of reduced fat Cheddar cheese. 1. Manufacture, composition, and yield. J. Dairy Sci. 77:3506-3515.

Moio, L., and F. Addeo. 1998. Grana Padano cheese aroma. J. Dairy Res. 65:317-333.

Molimard, P., and H. E. Spinnler. 1996. Review: Compounds involved in the flavor of surface mold-ripened cheeses: Origins and properties. J. Dairy Sci. 79:169-184.
SAS Institute. 2002. SAS for Windows. Release 9.0. SAS Institute Inc., Cary, NC.

Seon, K. H., J. Ahn, and H. S. Kwak. 2009. The accelerated ripening of cholesterol-reduced Cheddar cheese by crosslinked $\beta$-cyclodextrin. J. Dairy Sci. 92:49-57.

Ur-Rehman, S., J. M. Banks, E. Y. Brechany, D. D. Muir, P. L. H McSweeney, and P. F. Fox. 2000. Influence of ripening temperature on the volatiles profile and flavour of Cheddar cheese made from raw or pasteurized milk. Int. Dairy J. 10:55-65.

Walstra, P., T. J. Geurts, A. Noomen, A. Jellema, and M. A. J. S. van Boekel. 1999a. Dairy Technology: Principles of Milk Properties and Processes. Marcel Dekker Inc., New York, NY.

Walstra, P., A. Noomen, and T. J. Geurts. 1999b. Dutch-type varieties. Pages 39-82 in Cheese: Chemistry, Physics and Microbiology, Vol. 2: Major Cheese Groups. P. F. Fox, ed. Aspen Publishers, Gaithersburg, MD.

Wijesundera, C., and L. Drury. 1999. Role of milk fat in production of Cheddar cheese flavour using a fat-substituted cheese model. Aust. J. Dairy Technol. 54:28-35. 